\title{
Using Wearable Accelerometers in a Community Service Context to Categorize Falling Behavior
}

\author{
Chia-Hsuan Lee ${ }^{1}$, Tien-Lung Sun ${ }^{2, *}$, Bernard C. Jiang ${ }^{1}$ and Victor Ham Choi ${ }^{2}$ \\ 1 Department of Industrial Management, National Taiwan University of Science and Technology, No. 43, \\ Section 4, Keelung Road, Da'an District, Taipei 106, Taiwan; sweat0430@gmail.com (C.-H.L.); \\ bcjiang@mail.ntust.edu.tw (B.C.J.) \\ 2 Department of Industrial Engineering and Management, Yuan Ze University, 135 Yuan Tung Road, \\ Chungli District, Taoyuan 320, Taiwan; s1008909@mail.yzu.edu.tw \\ * Correspondence: tsun@saturn.yzu.edu.tw; Tel.: +886-3-463-8800 (ext. 2525)
}

Academic Editor: Anne Humeau-Heurtier

Received: 18 May 2016; Accepted: 5 July 2016; Published: 13 July 2016

\begin{abstract}
In this paper, the Multiscale Entropy (MSE) analysis of acceleration data collected from a wearable inertial sensor was compared with other features reported in the literature to observe falling behavior from the acceleration data, and traditional clinical scales to evaluate falling behavior. We use a fall risk assessment over a four-month period to examine $>65$ year old participants in a community service context using simple clinical tests, including the Short Form Berg Balance Scale (SFBBS), Timed Up and Go test (TUG), and the Short Portable Mental Status Questionnaire (SPMSQ), with wearable accelerometers for the TUG test. We classified participants into fallers and non-fallers to (1) compare the features extracted from the accelerometers and (2) categorize fall risk using statistics from TUG test results. Combined, TUG and SFBBS results revealed defining features were test time, Slope(A) and slope(B) in Sit(A)-to-stand(B), and range(A) and slope(B) in Stand(B)-to-sit(A). Of (1) SPMSQ; (2) TUG and SPMSQ; and (3) BBS and SPMSQ results, only range(A) in Stand(B)-to-sit(A) was a defining feature. From MSE indicators, we found that whether in the $X, Y$ or $Z$ direction, TUG, BBS, and the combined TUG and SFBBS are all distinguishable, showing that MSE can effectively classify participants in these clinical tests using behavioral actions. This study highlights the advantages of body-worn sensors as ordinary and low cost tools available outside the laboratory. The results indicated that MSE analysis of acceleration data can be used as an effective metric to categorize falling behavior of community-dwelling elderly. In addition to clinical application, (1) our approach requires no expert physical therapist, nurse, or doctor for evaluations and (2) fallers can be categorized irrespective of the critical value from clinical tests.
\end{abstract}

Keywords: multi-scale entropy; complexity; wearable accelerometers; falling; community service

\section{Introduction}

Mobility may be an important factor for fear of falling due to significantly high correlations [1]. Falling refers to the unexpected change in body position when the center of gravity is out of balance. The body is unable to respond effectively, and falls onto the floor or a lower place [2]. This issue has attracted an increased amount of attention as society ages. Aging degrades lower limb function and reduces the ability of the elderly to perform daily activities, even leading to increased probability of falls [3]. According to the study by [4], falling is the major cause of accidental death for people aged 65 and above. The body depends on the balancing mechanism to prevent falls related to inertial force of and on the body. For elderly, there is a gap between the physical response and self-expectation; self-awareness for falls is also low. Risk assessment and preventive measures for falls in the elderly will become an important area for developing care of aged populations [5]. Falling, besides causing 
death, may also induce disability and injuries to some degree [4]. The study of mobility of elderly [6,7] indicated that many falls have considerable relationships with movement disorder of elderly people (e.g., assess by standing up from the chair and walking ability). When summarizing fall risk factors, Rubenstein et al. [8] also suggested there is a strong relationship between falls and decreased muscle strength, followed by unsteady gait and balance disorder, which shows that declined mobility is the main cause of elderly people's falls.

The causes of falls are quite complex and include internal and external factors. For example, degradation of physical function is caused by aging, acute and chronic illnesses, drugs, and safety of the home environment, which are all related to fall occurrence. Typically, falls in the elderly are not caused by a single but rather many factors. Assessment and intervention from multiple aspects are required to effectively prevent the occurrence of falls and other related injuries [9]. To identify persons at risk of falling, thus being eligible for preventive treatment, many risk assessment tools, e.g., the 3-minute Timed Up and Go test (TUG) [10] or the Short Form Berg Balance Scale (SFBBS) [11] have been developed and evaluated in a multitude of studies. However, when medical professionals are performing assessments, if they not only use their professional knowledge to do the evaluation, but also include objective devices, this may allow them to include information such as user environment and user time. Evaluation can be performed and not limited to hospitals.

In addition to the above-mentioned clinical tests, wearable accelerometers are a viable technology for fall risk assessment, joining clinical and laboratory methods as acceptable assessment tools. Inertial-sensor-based systems have the benefits of portability, low cost, and few constraints on the types of movements that can be monitored [12]. Therefore, studies using wearable accelerometers can collect motion data for fall risk assessments. Tamura [13] designed a body worn accelerometer for fall detection suggesting falls occur at angles of inclination greater than 60 degrees. Kulkarni and Basu [14] discussed wearable tri-axial accelerometers based on fall detectors by presenting their types, mountings, and methods of detecting falls to minimize risk of injury. Marschollek et al. [15] developed an objective and unobtrusive method to determine individual fall risk based on the use of motion sensor data. Geriatric inpatients wore an accelerometer on the waist during a Timed Up and Go test and a $20 \mathrm{~m}$ walk; here, the wearable accelerometer was also used to monitor the physiological status of elderly in daily life [16]. Timed Up and Go (TUG) times have been associated with impaired mobility and an increased risk of falling [17]. TUG is an excellent indicator of clinically testing the walking ability of patients with musculoskeletal neurological system injury [10]. Accelerometers were the only inertial sensor in $70 \%$ of the studies, whereas gyroscopes were the only inertial sensor in one study [18]. Previous studies showed that tri-axial accelerometers are more accurate than other types of accelerometers $[10,19-23]$. We hope to use accelerometers to objectively record TUG in addition to clinical tests, thus providing multiple indicators of fall risk assessment. It is worth mentioning the clinical tests have a clear score boundary, as the distinction among and analyses and research behind accelerometers mostly reveal the factors or risks of falling using experiments. From a literature review, Howcroft et al. [12] suggested three main methods for classifying subjects into faller and non-faller categories: retrospective fall history $(30 \%)$, prospective fall occurrence $(15 \%)$, and scores on clinical assessments (32.5\%). Horak et al. [24] suggested, to be useful for clinicians, objective measures of balance and gait need to be available outside the laboratory, where recent advances in body-worn sensors have made this portability possible. Laboratory tests of gait and balance involve expensive, highly technical, non-portable equipment, such as video-based motion analysis systems and force plates, which are not practical for clinical environments or for multisite clinical trials [24]. Falls often occur during everyday life. In previous studies, people used well-controlled conditions to perform experiment investigation $[10,15,17-22]$. However, in real life, we cannot eliminate variables. We try to use an objective method to collect data and then perform indicative discussion. In this study, we try to discuss with respect to community services for the elderly in real world.

Previous studies discussing the feature of body-fixed accelerometers can provide insights into TUG performance [15,17,25-27]. Features included Sit-to-Stand, Stand-to-Sit durations, amplitude 
range (Range) and slopes (Jerk), mean step duration, step length, and number of steps during the TUG test. Acceleration median and standard deviation (SD) were also calculated. Further, Pincus et al. [28] used Approximate Entropy (ApEn) computed for accelerometer sensors. Entropy measures for time series, such as sample entropy (SampEn) and approximated entropy (ApEn) [29], do indeed measure the unpredictability (opposite of regularity) of a time series. More recently, Costa et al. [30,31] have proposed a new entropy-based measure for time series that seems to better quantify complexity, coining it Multiscale Entropy (MSE). Tsai [32] used MSE to measure the accelerometer, considering that multiscale entropy curves can be used to compare the differences between different statuses, i.e., when the body is under a relatively unbalanced status, the multiscale entropy curve will decrease. MSE can be used to quantify complexity on widely varying timescales; it is also worthwhile to explicitly compare the results of MSE used for time series analysis with classical characterizations of scaling and self-similarity. Signals with a higher level of complexity have greater self-similarity. In the context of biomedicine, greater physiological complexity indicates greater adaptability to the external environment; the reverse also holds true. This method is commonly used in the study of physiological signals and pathology $[31,33,34]$. In this study, due to the wearable accelerometer measure tri-axial signal being too messy [32], MSE is derived from the Approximate Entropy (ApEn) used by Pincus et al. [28]. In the past, Pincus et al. computed for sensors. Therefore, in addition to the analysis method using the accelerometer as described above, we try to use data from MSE analysis to quantify the balance of the body.

This study combines accelerometer sensing technology and clinical tests using MSE to analyze posture control ability to further discuss indicators of fall risk assessment. We also discuss what features of accelerometers outside the lab, e.g., within a community services context, can categorize fallers. The aim of this paper is to examine the MSE and sensor-based methods for fall risk assessment against conventional and established methods.

\section{Materials and Methods}

\subsection{General Approach}

We take a fall risk assessment screening service provided by a hospital in central Taiwan as an example based on four-month follow-up data (between 24 February and 18 May, 2015) obtained in a prospective study. The hospital staff, including rehabilitation doctors, physical therapists, and nurses, visited elderly community service centers in central Taiwan to advocate fall risk prevention concepts and conduct fall risk assessments. We tried to perform simple clinical tests on community-elderly in a fall prevention community service context, including BBS, TUG, SPMSQ, and allowed them to wear wearable accelerometers while performing the TUG test to compare data. In this study, due to the disorderly tri-axial signal of the wearable accelerometer [32], MSE is derived from Approximate Entropy (ApEn) as used by Pincus et al. [28]. In the past, Pincus et al. computed ApEn for sensors, but we try to use MSE analysis signals to quantify the balance status of the body. It is hoped that with the two groups of people who are divided based on the existing criteria of the clinical test, we can summarize the $t$-test to explore whether the groups sorted by the accelerometer feature and MSE also have the ability to distinguish and sort two groups. Further, we wish to understand how wearable accelerometers in a community service context could be used to indicate and assess fall risks. This study was approved by the Institutional Review Board of Tsaotun Psychiatric Center, Ministry of Health and Welfare.

\subsection{Study Population}

A total of 65 elderly individuals were recruited as a convenience sample. According to the professional team, the enrolment criteria of the subjects were as follows: no musculoskeletal system injuries, no history of central nervous system injuries, able to walk independently with or without the use of any aids within the last three months. After the researcher explained the details, each subject 
agreed to participate and signed the consent forms prior to entering the laboratory. Table 1 lists the details of the participants.

Table 1. Characteristics of the community-dwelling elderly.

\begin{tabular}{cccc}
\hline & & Number & Average \pm SD \\
\hline \multirow{2}{*}{ Gender } & Female & 49 & $77 \pm 6.60$ \\
& Male & 16 & $73 \pm 6.00$ \\
\hline \multirow{3}{*}{ Age } & $65-70$ & 12 & $69 \pm 1.72$ \\
& $71-75$ & 25 & $73 \pm 1.53$ \\
& $76-80$ & 12 & $78 \pm 1.48$ \\
& $80+$ & 16 & $85 \pm 4.60$ \\
\hline
\end{tabular}

\subsection{Clinical Test}

There are many factors causing elderly people's falls. To raise the participation rate of the elderly and to understand different aspects of the impact, this study used three kinds of quick and simple clinical assessment scales for the tests, including the Short Form Berg Balance Scale (SFBBS) [11], Timed Up and Go test (TUG) [10], Short Portable Mental Status Questionnaire (SPMSQ) [35]. Details of each clinical scale are as follows:

- Short Form Berg Balance Scale (SFBBS) is used to assess balance. It is a simplified version of the Berg Balance Scale (BBS) [36] and contains seven activities: (a) getting in and out of a chair, sitting unsupported, and transferring from a bed to a chair; (b) continuous standing with feet together, feet apart, and with eyes closed; (c) turning to each side and turning 360 degrees; (d) reaching forward; (e) picking up an object from the floor; (f) tandem and unilateral stance; and (g) dynamic weight shifting. Compared to BBS, only half the time (about $10 \mathrm{~min}$ ) was required to complete all activities. This scale scored individuals based on their performance in each activity: inability to perform the activity scored 0 points, inability to complete $50 \%$ of the activity scored 2 points, and ability to complete the activity scored 4 points, with a total score of 28 points.

- $\quad$ Timed Up and Go test (TUG) is used to evaluate gait ability. The TUG test is a well-known clinical test of mobility and fall risk [17]. It is a commonly used screening tool for falls risk in the inpatient and community setting [37]. The Up and Go test, a predecessor of the TUG was introduced by Mathias et al. [38], where multiple components of the test are scored by an observer. Podsiadlo and Richardson [10] introduced the timed version of the test; for the assessment, chair legs are aligned with the reference line starting point and a triangular pyramid is placed $3 \mathrm{~m}$ in front of the reference line. The subjects are asked to sit on the chair with knees naturally bent and are not allowed to place the feet on or outside the reference line. The subjects are then asked to stand up in a comfortable way, walk straight ahead for 3 meters, then return to the original place and sit down. In this study, we used the timed version to enhance the objectivity of the test.

- The Short Portable Mental Status Questionnaire (SPMSQ) focuses on cognitive functions. This scale has 10 questions from six dimensions to evaluate consciousness, memory, orientation, attention, thinking process, and general knowledge, to obtain a preliminary understanding of the state of mental health of the elderly individual. Simple mental status questionnaires are conducted and the measuring method is simple. The elderly individual can either complete the questionnaires on their own or ask for help from their family members to perform a preliminary screen for dementia. A clinical diagnosis of dementia risk is when an individual has incorrectly answered three or more questions. Fischer et al. [39] used SPMSQ and found that declining cognition is associated with the performance of mobility activities in an unsafe manner, thereby increasing the risk of falling. Lin et al. [40] also used SPMSQ for falls among community-dwelling elderly assessed in annual geriatric health examinations. 


\subsection{Wearable Accelerometer}

For the community service aspect of the study, all participants wore a wireless tri-axial accelerometer system (Freescale RD3152MMA7260Q, Freescale Semiconductor-NXP, Austin, TX, USA) on a belt around the waist (Figure 1a) during the TUG test. We tried to place the accelerometer on the lower back, including the pelvis, sacrum, and the L3 to L5 vertebrae (Figure 1b), which is the most common sensor location and was the only possible location for $65 \%$ of the studies [12]. This site approximates the center of mass location. The data were transmitted to a PC and stored for processing.

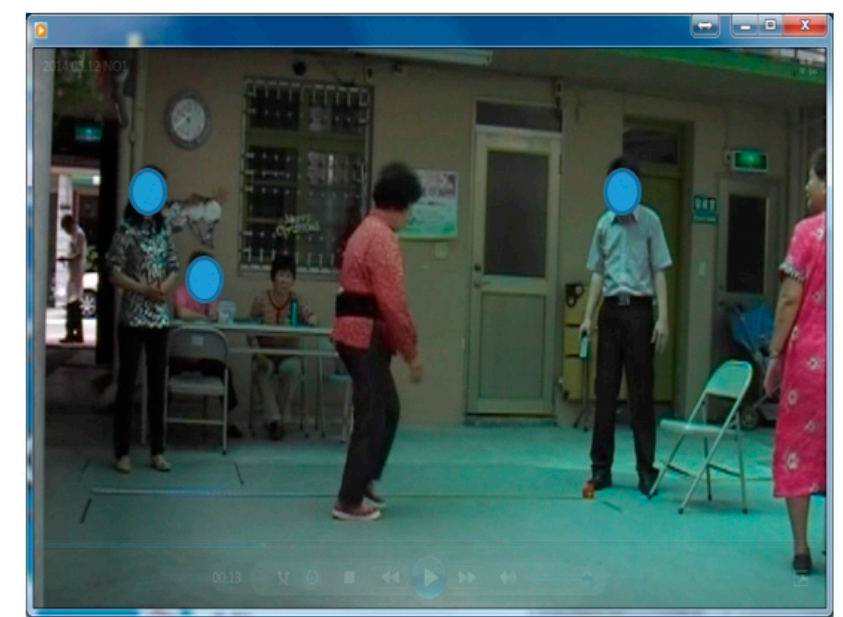

(a)

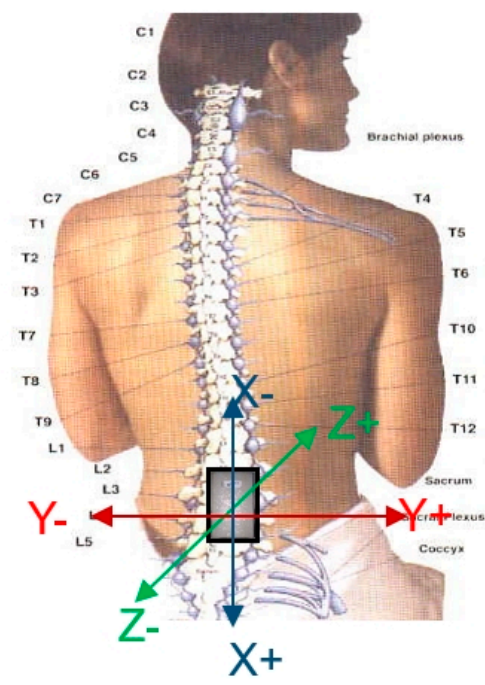

(b)

Figure 1. Wearable accelerometer (a) put on a belt around the waist (b) location.

\subsection{Features Extracted from Accelerometers}

The TUG test is a widely used clinical measure of mobility and fall risk in older adults. Weiss et al. [17] proved that body-fixed accelerometers can provide insight into TUG performance. Sit-to-Stand and Stand-to-Sit times were extracted from the anterior-posterior (AP) signal slopes. Sit-to-Stand and Stand-to-Sit slopes seemed to break into two different slopes in the middle of each Sit-to-Stand and Stand-to-Sit time interval. Therefore, we divided the Sit-to-Stand and Stand-to-Sit intervals into two equal parts. Parameters included Sit(A)-to-Stand(B), Stand(B)-to-Sit(A) durations, amplitude range (Range), and slopes (Jerk). Acceleration median and standard deviation (SD) were also calculated. The outcome measures definitions are shown in Figure 2 and the features descriptions are outlined below:

- Slope (Jerk): Linear fit of the acceleration data in the Sit-to-Stand and Stand-to-Sit stages of the TUG and represents the rate of change in acceleration.

- Range: Difference between the maximum and minimum acceleration values in the Sit-to-Stand or the Stand-to-Sit aspect of the TUG.

- Time: Sit-to-Stand and Stand-to-Sit accelerometer derived duration.

- Statistics: Sit-to-Stand and Stand-to-Sit mean, median, and standard deviation (SD).

When explaining our segmentation points, we recommend Moe-Nilssen's [41] calibration algorithm, which transforms the data to the horizontal-vertical coordinate system. Figure 3a shows an example of the acceleration signal in a young, healthy adult as he performed a Sit-to-Stand, followed by quiet standing, then a Stand-to-Sit [17]. In our study, we categorize these definitions in Table 2. In this work, interval 1-2 was examined with reference to the Sit-to-Stand task and interval 5-6 was examined with reference to the Stand-to-Sit task. 


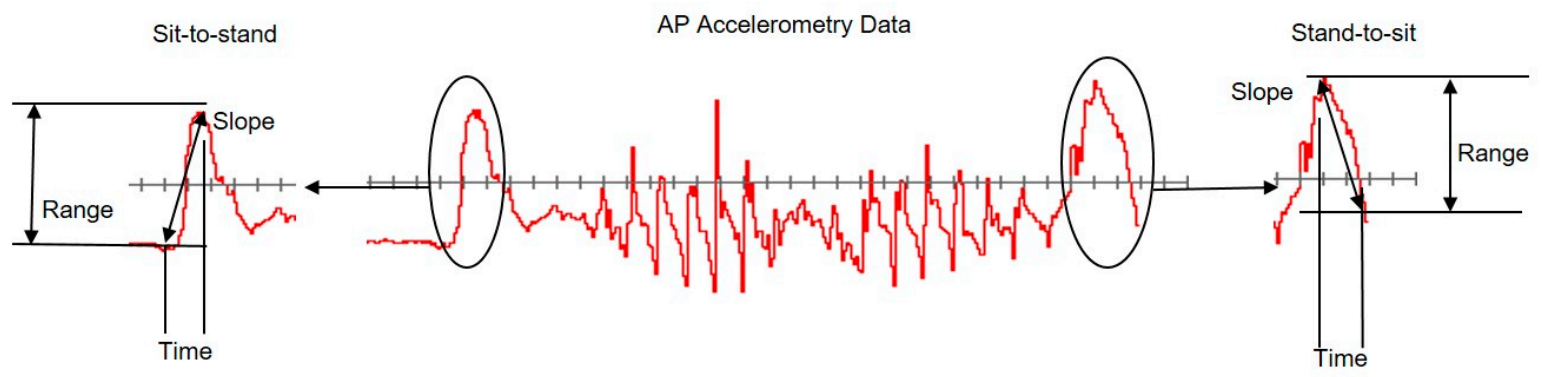

Figure 2. The outcome measures definitions.

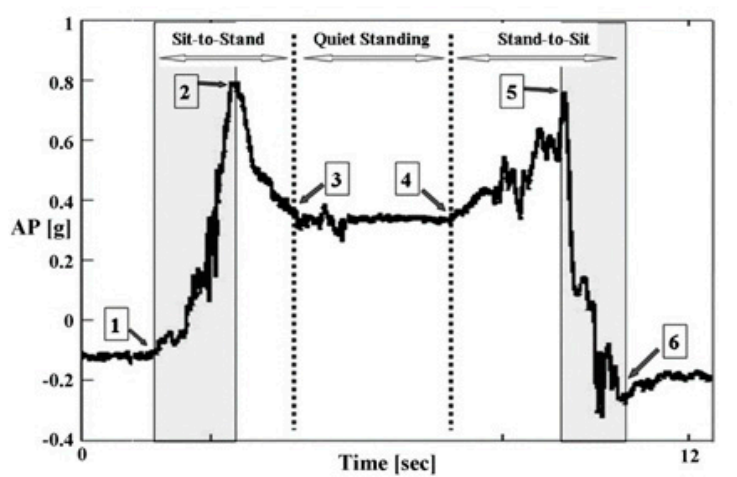

(a)

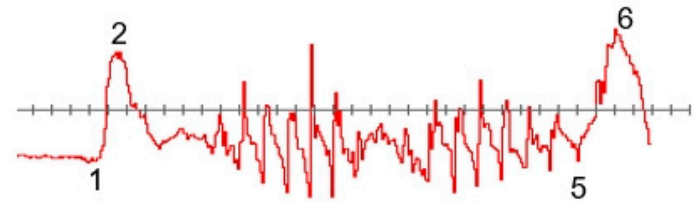

(b)

Figure 3. Segmentation points. (a) The anterior-posterior (AP) signal during Sit-to-Stand, followed by quiet standing and then by Stand-to-Sit, in a young healthy control subject [17]; (b) Our study's segmentation points.

Table 2. Segmentation points and descriptions.

\begin{tabular}{cl}
\hline Point & \multicolumn{1}{c}{ Description } \\
\hline 1 & The min. AP acceleration peak just before the signal starts to rise from steady state \\
2 & First "M-like" AP acceleration maximum peak \\
3 & Difficult to reliably detect (first step) because it is inconsistent among patients \\
4 & Same as No. 3 (last turn) \\
5 & The second "M-like" AP acceleration maximum peak \\
6 & The minimum acceleration peak right when the acceleration reaches steady state \\
\hline
\end{tabular}

Marschollek et al. [15] investigated the use of wearable accelerometers to provide objective data on motion features and automatically assessed individual fall risk. A sensor-based assessment of fall risk was performed, which employs gait and motion parameters obtained during a TUG test and a $20 \mathrm{~m}$ walk. These features are as follows: mean step duration, step length, number of steps, and statistics during the TUG test. The feature descriptions are show below. We try to use these features to assess subject performance.

- No. of steps and average step length: Gait regularity variables can discriminate between faller and non-faller populations. The analysis of gait regularity is pertinent in early detection of gait degradation.

- $\quad$ Statistics: Mean (Mean AP), SD (standard deviation AP) calculated from points 1-6.

\subsection{Multiscale Entropy (MSE)}

The MSE approach, proposed by Costa [30] in 2002, has now been effectively applied in many fields; there have been numerous cases where it was applied to physiological signals. The MSE 
calculation process has three steps: the coarse-graining process, the sample entropy, and the complexity index. The calculation process of this method is described as follows.

- Coarse-graining process: the coarse-graining process is intended to divide time series into different time scales to calculate entropy for different time and space scales. Figure 4 shows the process of coarse-graining. $x_{i}$ is the original time series data point; Scale 1 is the original time series; Scale 2 is the average of two points on the original time series, which becomes the time series of Scale 2, and so on. The calculation formula of each scale is shown as formula (1), with y being the data point, $\tau$ being the scale of segmentation, and $N$ being the size of the original dataset.

$$
y_{j}^{(\tau)}=\frac{1}{\tau} \sum_{i=(j-1) \tau+1}^{j \tau} x_{i} 1 \leqslant j \leqslant \frac{N}{\tau}
$$

- Sample Entropy: After using of Coarse-graining process to obtain time series for different time scales, you can calculate the time series individually for sample entropy. The sample entropy calculation process is as follows:

Step 1: Set the data comparison number $m$ and threshold value $r$. $m$ is usually set to 2 or 3 , while $r$ is usually set at 0.25 .

Step 2: Take $m \mathrm{X}$ data points in the time series data as the comparison benchmark. For example, for time series $\mathrm{X}=\left(x_{1}, \ldots, x_{7}\right)$, when data comparison number $m=2$, the comparison unit group is $\left\{\left(x_{1}, x_{2}\right),\left(x_{2}, x_{3}\right),\left(x_{3}, x_{4}\right),\left(x_{4}, x_{5}\right),\left(x_{5}, x_{6}\right),\left(x_{6}, x_{7}\right)\right\}$. For the first time compare $\left(x_{1}\right.$, $\left.x_{2}\right)$ with the other five unit groups. For the second time point, compare $\left(x_{2}, x_{3}\right)$ with the other four unit groups.

Step 3: When forming comparisons, calculate the maximum difference in the comparison group. The calculation method follows formula (2):

$$
d\left[x_{i}, x_{j}\right]=\max \left[\left|x_{i+k-1}-x_{j+k-1}\right|\right], 1 \leqslant k \leqslant m, i \leqslant N-m, j \leqslant N-m
$$

Step 4: Compare the calculated d $\left[x_{i}, x_{j}\right]$ with $r \times S$. where $\mathrm{S}$ is the standard difference of original time series data point. If $\mathrm{d}\left[x_{i}, x_{j}\right]$ is smaller than $r \times S$, then the two comparison groups are similar. In addition, add 1 to the accumulated similar number $n_{i(m)}$, and calculate the occurrence probability of similar number $\mathrm{C}_{i(m)}$ after all comparison processes are completed. The calculation method follows formula (3).

$$
C_{i(m)}=\frac{n_{i(m)}}{N-m}, \quad 1 \leqslant i \leqslant N-m
$$

Step 5: Return to step 1 and change the original comparison number $m$ to $m+1$, repeat step 2 to step 3 and obtain the accumulated similar number $n_{i(m)}$ and the occurrence probability of the similar number $C_{i(m+1)}$. The calculation method follows formula (4):

$$
C_{i(m+1)}=\frac{n_{i(m+1)}}{N-m-1}, \quad 1 \leqslant i \leqslant N-m-1
$$

Step 6: Take the average of all occurrence probabilities of the similar number $C_{i(m)}$ with the comparison number $\mathrm{m}$ as the denominator, take the average of the occurrence probability of the similar number $C_{i(m+1)}$ with the comparison number $m+1$ as the numerator, and take the natural logarithm and negative value as the sample entropy. The calculation formula follows (5):

$$
\text { Sample Entropy }(m, r, N)=-\ln \left(\frac{\sum C_{i(m+1)} / N-m-1}{\sum C_{i(m)} / N-m}\right)
$$


- Complexity Index (CI): We can plot SampEn as a function of the scale factor to calculate the area under the CI, as shown in Figure 5. The grey area under the black curve represents the complexity Index (CI). The calculation method follows formula (6):

$$
\text { Complexity Index }=\sum_{i=1}^{N} \text { Sample Entropy }(i)
$$

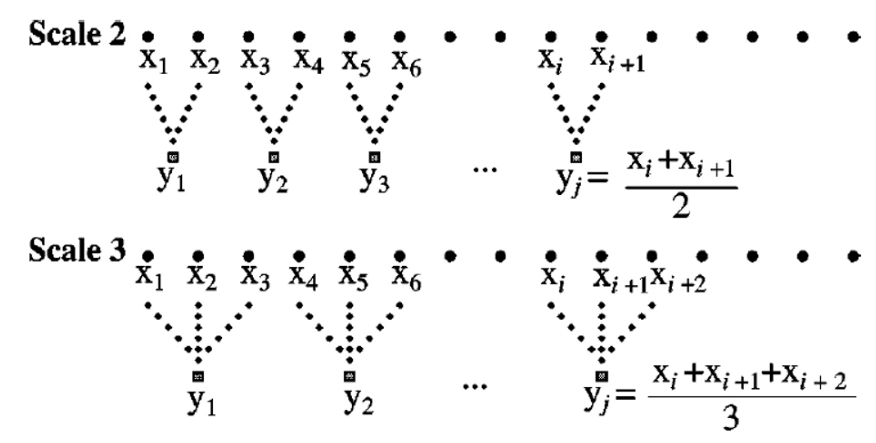

Figure 4. Coarse-graining process [30].

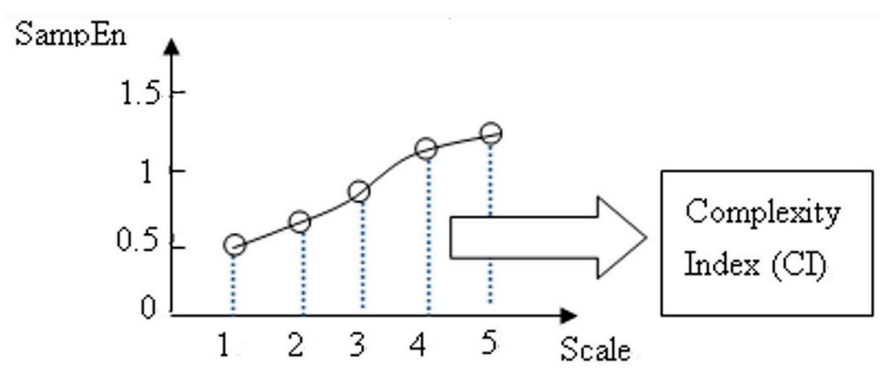

Figure 5. Schematic illustration of the definition of the complexity index.

\section{Results and Discussion}

Our discussion and analysis can be divided into three main parts. (a) We simply use a clinical test to determine who has fall risk in order to categories the people with and without a fall risk (this includes efficiency); (b) We use the results in (a) to compare accelerometer features, and then use $t$-test analysis to verify the categorization of fall risk; (c) Finally, we use the results from (a) to compare the MSE results, and we then use t-test analysis to verify the categorization of fall risk. Since scales of clinical tests have clear boundary scores, Karthikeyan et al. [42], Kim et al. [43], İlçin et al. [44] and Li et al. [45] proposed that balance is considered as impaired when the score is 23 or below for BBS. Barry et al. [37], Shumway-Cook et al. [46], Lindsay et al. [47] and Kwoka et al. [48] recommend that it is considered a high risk if the time for TUG is greater than $13.5 \mathrm{~s}$. Furthermore, in clinical judgments of the SPMSQ, there may be a risk of dementia if a subject answered three or more questions incorrectly in the test. We try to use these indicators as judgment criteria for categorizing fallers and non-fallers. Table 3 shows the results of the fall risk assessment tests for predicting fall events from a community service context.

We then used the results from the clinical indicators to compare the features extracted from the accelerometer (Section 2.5) and distinguished elderly people with fall risk using statistics from the significance of the TUG test result. Since previous publications have performed discussion and verification on the clinical test score (according to fallers and non-fallers), we try to divide each clinical test into two groups. When comparing accelerometer features with the t-test, a $p$-value smaller than 0.05 implies a statistically significant difference, indicating fallers distinguished by features are 
quite similar to the clinical test result. For example, in Table 4, two groups of people from TUG are assigned an overall steps feature, and the t-test was used for verification. The $p$-value is 0.044 , which shows significant difference. Thus, this feature is able to distinguish people with fall risk. As indicated by Table 2, in terms of TUG, the more defining features are steps, average step length, test time, Slope(A), time(A) and slope(B) in Sit(A)-to-Stand(B), and range(A), slope(B), and time(B) in Stand(B)-to-Sit(A). Regarding BBS, the more defining features are test time, Slope(A), time(A) and slope(B) in Sit(A)-to-Stand(B), and range(A) and slope(B) in Stand(B)-to-Sit(A). Regarding the SPMSQ, only range(A) in Stand(B)-to-Sit(A) are defining features. If we look at the screening results of both TUG and BBS, the defining features are test time, slope(A) and slope(B) in Sit(A)-to-Stand(B), and range(A) and slope(B) in Stand(B)-to-Sit(A). Looking at the screening results of TUG and SPMSQ, only range(A) in Stand(B)-to-Sit(A) is a defining feature, which is the same result for the SPMSQ. These results suggest that slope(B) in Stand-to-Sit is the most defining feature. Fallers can be effectively categorized irrespective of the critical value used in the clinical test. From MSE indicators, we found that whether in the X, Y or Z direction, TUG, BBS, and combined TUG and BSS are all distinguishable, showing MSE can effectively classify participants in these clinical tests using behavioral actions.

Table 3. Clinical indicators used to categorize classified fallers and non-fallers.

\begin{tabular}{cl}
\hline Clinical Test & \multicolumn{1}{c}{ Faller No. } \\
\hline TUG & $19,23,25,26,32,35,36,37,38,39,40,46$ \\
BBS & $4,19,22,25,32,35,36,39,40,46,67,70$ \\
SPMSQ & $19,36,39,51,61,67$ \\
TUG and BBS & $19,25,32,35,36,39,40,46$ \\
TUG and SPMSQ & $19,36,39$ \\
BBS and SPMSQ & $19,36,39,67$ \\
\hline
\end{tabular}

Table 4. Critical $p$-values from the clinical tests used to distinguish accelerometer features.

\begin{tabular}{|c|c|c|c|c|c|c|c|}
\hline \multicolumn{2}{|c|}{ Accelerometer Features } & \multicolumn{6}{|c|}{ Clinical Test } \\
\hline & & TUG & BBS & $\begin{array}{c}\text { TUG } \\
\text { and BBS }\end{array}$ & SPMSQ & $\begin{array}{l}\text { TUG and } \\
\text { SPMSQ }\end{array}$ & $\begin{array}{l}\text { BBS and } \\
\text { SPMSQ }\end{array}$ \\
\hline \multirow{5}{*}{ Overall } & Steps & 0.044 * & 0.088 & 0.118 & 0.399 & 0.196 & 0.147 \\
\hline & Average steps length & $0.003 *$ & 0.307 & 0.129 & 0.362 & 0.127 & 0.094 \\
\hline & Test time & $0.000 *$ & 0.004 * & 0.001 * & 0.263 & 0.019 * & 0.177 \\
\hline & Mean AP & 0.057 & 0.121 & 0.082 & 0.483 & 0.148 & 0.135 \\
\hline & standard deviation $\mathrm{AP}$ & 0.101 & 0.113 & 0.238 & 0.405 & 0.437 & 0.100 \\
\hline \multirow{9}{*}{ Sit(A)-to-Stand(B) } & Slope(A) & $0.012 *$ & 0.011 * & $0.044^{*}$ & 0.107 & 0.130 & 0.085 \\
\hline & Range(A) & 0.117 & 0.168 & 0.374 & 0.255 & 0.395 & 0.150 \\
\hline & Time(A) & $0.022 *$ & $0.018^{*}$ & 0.055 & 0.219 & 0.182 & 0.195 \\
\hline & Slope(B) & $0.001 *$ & $0.006^{*}$ & $0.007^{*}$ & 0.118 & 0.011 * & 0.250 \\
\hline & Range(B) & 0.067 & 0.226 & 0.361 & 0.188 & 0.430 & 0.375 \\
\hline & Time(B) & 0.100 & 0.153 & 0.125 & 0.216 & 0.362 & 0.276 \\
\hline & Mean & 0.273 & 0.320 & 0.241 & 0.358 & 0.199 & 0.382 \\
\hline & Median & 0.291 & 0.163 & 0.288 & 0.261 & 0.051 & 0.382 \\
\hline & standard deviation & 0.233 & 0.368 & 0.402 & 0.345 & 0.394 & 0.387 \\
\hline \multirow{9}{*}{ Stand(B)-to-Sit(A) } & Slope(A) & 0.190 & 0.226 & 0.450 & 0.177 & 0.443 & 0.484 \\
\hline & Range(A) & $0.006^{*}$ & 0.0137 * & $0.006^{*}$ & 0.180 & 0.086 & 0.168 \\
\hline & Time(A) & 0.073 & 0.155 & 0.231 & 0.294 & 0.355 & 0.311 \\
\hline & Slope(B) & $0.002 *$ & 0.0229 * & 0.033 * & 0.014 * & 0.022 * & $0.043 *$ \\
\hline & Range(B) & 0.313 & 0.313 & 0.500 & 0.096 & 0.253 & 0.235 \\
\hline & Time(B) & $0.014^{*}$ & 0.052 & 0.060 & 0.310 & 0.216 & 0.261 \\
\hline & Mean & 0.267 & 0.192 & 0.293 & 0.313 & 0.161 & 0.097 \\
\hline & Median & 0.401 & 0.209 & 0.421 & 0.269 & 0.185 & 0.077 \\
\hline & standard deviation & 0.319 & 0.184 & 0.391 & 0.101 & 0.222 & 0.202 \\
\hline
\end{tabular}

* Significant values: $p$-value $<0.05$.

In general, distinguishing global features does not appear as effective as Sit(A)-to-Stand(B) and Stand(B)-to-Sit(A). As shown in the TUG task, compared to the sit and stand motion, walking is less effective in determining fall risks, which corroborates with the findings of Weiss et al. [17]—relevant 
and useful information lies in the acceleration signal of the TUG, especially in the Sit-to-Stand and Stand-to-Sit intervals. In general, distinguishing slope and range is more effective, which accords with the previous discussion of fallers and non-fallers from acceleration-derived measures by the TUG test that differed in the two groups [17,49,50]; among these studies, slope and range are the features showing differences between the two groups. It appears that these TUG features are common among diverse groups of fallers. To the best of our knowledge, we report here for the first time on the application of accelerometer-based measures that systematically evaluate TUG performance in a community service context, focusing on the Sit-to-Stand and Stand-to-Sit transitions. Looking at the screening results of BBS and SPMSQ, only range(A) in Stand(B)-to-Sit(A) is a defining feature.

Further, we also used MSE for comparisons and analyses. As shown in Table 5, we found by categorizing fallers and non-fallers by critical values from clinical tests using MSE. MSE has previously been used to detect the complexity of physiological signals, such as heart beat $[30,51,52]$ brain waves [53], acceleration [32], and postural stability [52,54]. The results all showed that MSE can effectively identify objective data. The results of this study, compared with the subjective SPMSQ survey, show MSE is most effective under tasks with behavioral actions (TUG, BBS). In addition, past studies using MSE analyzed postural stability mostly with tasks under experimental environments, such as opening eyes with both feet planted vs. opening eyes with a single foot planted [55], quiet standing vs. dual tasking [56], or subject type, such as elderly vs. young man (or patient vs. non-patient) [57-59]. Therefore, the tasks were used to compare the difference in complexity level. However, we used MSE in this study to analyze data collected in a non-controlled community service environment, but there was no difference in test type and experimental task. The results show that it can also effectively distinguish fallers and non-fallers, indicating that the acceleration collected by MSE using the accelerometer is also an effective defining feature.

Table 5. Critical $p$-values from the clinical tests used to distinguish accelerometers through Multiscale Entropy (MSE) analysis.

\begin{tabular}{ccccccc}
\hline & \multicolumn{5}{c}{ Clinical Test } \\
\cline { 2 - 6 } & TUG & BBS & BBS and TUG & SPMSQ & TUG and SPMSQ & BBS and SPMSQ \\
\hline MSE-X & $0.002^{*}$ & $0.014^{*}$ & $0.006^{*}$ & $0.031^{*}$ & 0.106 & 0.133 \\
MSE-Y & $0.006^{*}$ & $0.020^{*}$ & $0.012^{*}$ & 0.442 & $0.011^{*}$ & 0.184 \\
MSE-Z & $0.013^{*}$ & $0.048^{*}$ & $0.036^{*}$ & 0.072 & $0.033^{*}$ & 0.299 \\
\hline
\end{tabular}

* Significant values: $p$-value $<0.05$.

We can conclude by our preliminary results that recording TUG using a wearable accelerometer and simultaneously providing quantifiable analysis and more effective defining features in real life provides objective clinical reference data. As mentioned by Weiss et al. [17], compared to the traditional method of using a stopwatch to distinguish fallers from non-fallers, we obtained significant results using TUG durations derived from the acceleration signal. To the best of our knowledge, we report for the first time using MSE for applying accelerometer-based measures that systematically evaluate TUG performance in a community service context compared with other clinical tests. As this is an inaugural study using MSE analysis with accelerometers, we attempt to prove that this analysis method is effective in categorizing fallers and non-fallers.

\section{Conclusions}

Previous studies mostly used wearable accelerometers to discuss group difference or detect performance of experimental tasks. In this paper, the MSE analysis of acceleration data collected from wearable inertial sensor was compared with (1) other features reported in the literature to observe falling behavior from the acceleration data; and (2) traditional clinical scales to evaluate falling behavior. The results indicated that MSE analysis of acceleration data can be used as an effective metric to categorize falling behavior of community-dwelling elderly. We hope this study indicates 
through current results that an ordinary and low cost method can be available outside the laboratory and advances in body-worn sensors have recently made this portability possible. From this study, we have seen evidence that, in addition to clinical application value, the advantage of our approach is not needing an expert physical therapist, nurse, or doctor for evaluations. In the future, we recommend increasing the age scope in order to distinguish the difference between each age group. In addition, we can use different entropy analysis methods, such as the multivariate multiscale entropy (MMSE), to perform interpretations or to increase different aspects of the clinical test in order to perform follow-up investigation so that body-worn sensors can effectively be introduced for everyone to use.

Acknowledgments: The researchers would like to thank the staff of the Feng-Yuan Hospital, Taiwan. This study is supported by the Ministry of Science and Technology under project NSC103-2221-E-011 -122 -MY3 and MOST 103-2221-E-155 -044-MY3, for which we are especially grateful.

Author Contributions: Chia-Hsuan Lee, Tien-Lung Sun and Bernard C. Jiang designed the study. Chia-Hsuan Lee and Victor Ham Choi was responsible for data collection and analysis. Chia-Hsuan Lee, Tien-Lung Sun, Bernard C. Jiang and Victor Ham Choi reviewed relevant literature and interpreted the acquired data. Chia-Hsuan Lee drafted the manuscript. All authors have read and approved the final manuscript.

Conflicts of Interest: The authors declare no conflict of interest.

\section{References}

1. Lin, Y. Relative Contribution of Mobility and Cognition to Falls and Fear of Falling in Cognitive Impaired Older Adults. Master's Thesis, National Taiwan University, Taipei, Taiwan, 2014.

2. Tinetti, M.E.; Speechley, M.; Ginter, S.F. Risk factors for falls among elderly persons living in the community. N. Engl. J. Med. 1988, 319, 1701-1707. [CrossRef] [PubMed]

3. Cheng, I.C. Biomechanical Comparisons of Lower Limb Muscle Power among Healthy Elderly, Falling Elderly and Young Adults during Performing Sit-to-Stand and Squatting Movement. Master's Thesis, National Yang-Ming University, Taipei, Taiwan, 2008.

4. Sattin, R.W.; Lambert Huber, D.A.; Devito, C.A.; Rodriguez, J.G.; Ros, A.; Bacchelli, S.; Stevens, J.A.; Waxweiler, R.J. The incidence of fall injury events among the elderly in a defined population. Am. J. Epidemiol. 1990, 131, 1028-1037. [PubMed]

5. Luo, Z.Q. Design and Development of a Portable Fall Risk Assessment System. Master's Thesis, Southern Taiwan University, Tainan, Taiwan, 2012.

6. Graafmans, W.C.; Ooms, M.E.; Hofstee, H.M.A.; Bezemer, P.D.; Bouter, L.M.; Lips, P. Falls in the elderly: A prospective study of risk factors and risk profiles. Am. J. Epidemiol. 1996, 143, 1129-1136. [CrossRef] [PubMed]

7. Kiely, D.K.; Kiel, D.P.; Burrows, A.B.; Lipsitz, L.A. Identifying nursing home residents at risk for falling. J. Am. Geriatr. Soc. 1998, 46, 551-555. [CrossRef] [PubMed]

8. Rubenstein, A.J.; Langlois, J.H.; Roggman, L.A. What Makes a Face Attractive and Why: The Role of Averageness in Defining Facial Beauty. In Facial Attractiveness, Advances in Visual Cognition; Rhodes, G., Zebrowitz, L., Eds.; Ablex: New York, NY, USA, 2002; Volume 1, pp. 1-33.

9. Li, Z.Y.; Lu, F.P.; Chan, D.C. Risk Factors, Evaluation and Prevention of Falls in Older Adults. Taiwan Soc. Intern. Med. 2014, 25, 137-142.

10. Podsiadlo, D.; Richardson, S. The Timed “Up \& Go": A Test of Basic Functional Mobility for Frail Elderly Persons. J. Am. Geriatr. Soc. 1991, 39, 142-148. [PubMed]

11. Chou, C.Y.; Chien, C.W.; Hsueh, I.P.; Sheu, C.F.; Wang, C.H.; Hsieh, C.L. Developing a short form of the Berg Balance Scale for people with stroke. Phys. Ther. 2006, 86, 195-204. [PubMed]

12. Howcroft, J.; Kofman, J.; Lemaire, E.D. Review of fall risk assessment in geriatric populations using inertial sensors. J. Neuroeng. Rehabil. 2013, 10. [CrossRef] [PubMed]

13. Tamura, T. Wearable accelerometer in clinical use. In Proceedings of 27th Annual International Conference of the IEEE Engineering in Medicine and Biology Society, Shanghai, China, 1-4 September 2005.

14. Kulkarni, S.; Basu, M.A. Review on Wearable Tri-Axial Accelerometer Based Fall Detectors. J. Biomed. Eng. Technol. 2013, 1, 36-39.

15. Marschollek, M.; Rehwald, A.; Wolf, K.H.; Gietzelt, M.; Nemitz, G.; Meyer, Z.; Schwabedissen, H.; Haux, R. Sensor-based fall risk assessment-an expert 'to go'. Methods Inf. Med. 2011, 50, 420-426. [CrossRef] [PubMed] 
16. Lee, Y.C. The Study of Physical Activity Evaluation for Elderly People. Master's Thesis, National Yang-Ming University, Taipei, Taiwan, 2010.

17. Weiss, A.; Herman, T.; Plotnik, M.; Brozgol, M.; Maidan, I.; Giladi, N.; Gurevich, T.; Hausdorff, J.M. Can an accelerometer enhance the utility of the Timed Up \& Go Test when evaluating patients with Parkinson's disease? Med. Eng. Phys. 2010, 32, 119-125. [PubMed]

18. Najafi, B.; Aminian, K.; Loew, F.; Blanc, Y.; Robert, P.A. Measurement of stand-sit and sit-stand transitions using a miniature gyroscope and its application in fall risk evaluation in the elderly. IEEE Trans. Biomed. Eng. 2002, 49, 843-851. [CrossRef] [PubMed]

19. Godfrey, A.; Conway, R.; Meagher, D.; Ólaighin, G. Direct measurement of human movement by accelerometry. Med. Eng. Phys. 2008, 30, 1364-1386. [CrossRef] [PubMed]

20. Culhane, K.M.; O'Connor, M.; Lyons, D.; Lyons, G.M. Accelerometers in rehabilitation medicine for older adults. Age Ageing 2005, 34, 556-560. [CrossRef] [PubMed]

21. Bourke, R.; Holden, B.; Curzon, J. Using Evidence to Challenge Practice: A Discussion Paper; Ministry of Education: Wellington, New Zealand, 2005.

22. Mathie, M.J.; Coster, A.C.; Lovell, N.H.; Celler, B.G. Accelerometry: Providing an integrated, practical method for long-term, ambulatory monitoring of human movement. Physiol. Meas. 2004, 25, R1-R20. [CrossRef] [PubMed]

23. Welk, G.J.; Schaben, J.A.; Morrow, J.R. Reliability of accelerometry-based activity monitors: A generalizability study. Med. Sci. Sports Exerc. 2004, 36, 1637-1645. [PubMed]

24. Horak, F.B.; Mancini, M. Objective biomarkers of balance and gait for Parkinson's disease using body-worn sensors. Mov. Disord. 2013, 28, 1544-1551. [CrossRef] [PubMed]

25. Bautmans, I.; Jansen, B.; van Keymolen, B.; Mets, T. Reliability and clinical correlates of 3D-accelerometry based gait analysis outcomes according to age and fall-risk. Gait Posture 2011, 33, 366-372. [CrossRef] [PubMed]

26. Caby, B.; Kieffer, S.; Hubert, M.S.; Cremer, G.; Macq, B. Feature extraction and selection for objective gait analysis and fall risk assessment by accelerometry. Biomed. Eng. Online 2011, 10. [CrossRef] [PubMed]

27. Ishigaki, N.; Kimura, T.; Usui, Y.; Aoki, K.; Narita, N.; Shimizu, M.; Hara, K.; Ogihara, N.; Nakamura, K.; Kato, H.; et al. Analysis of pelvic movement in the elderly during walking using a posture monitoring system equipped with a triaxial accelerometer and a gyroscope. J. Biomech. 2011, 44, 1788-1792. [CrossRef] [PubMed]

28. Pincus, S.M. Approximate entropy as a measure of system complexity. Proc. Natl. Acad. Sci. USA 1991, 88, 2297-2301. [CrossRef] [PubMed]

29. Pincus, S.M.; Goldberger, A.L. Physiological time-series analysis: What does regularity quantify? Am. J. Physiol. 1994, 266, H1643-H1656. [PubMed]

30. Costa, M.; Goldberger, A.L.; Peng, C.K. Multiscale Entropy Analysis of Complex Physiologic Time Series. Phys. Rev. Lett. 2002, 89, 068102. [CrossRef] [PubMed]

31. Costa, M.; Goldberger, A.L.; Peng, C.K. Multiscale entropy analysis of biological signals. Phys. Rev. E 2005, 71, 021906. [CrossRef] [PubMed]

32. Tsai, S.C. Analysis of Postural Sway Dynamics Using Multi Scale Entropy and Iterative Gaussian Filter. Master's Thesis, National Taiwan Normal University, Taipei, Taiwan, 2010.

33. Rosso, O.A.; Martin, M.T.; Plastino, A. Brain electrical activity analysis using wavelet-based informational tools (II): Tsallis non-extensivity and complexity measures. Phys. A 2003, 320, 497-511. [CrossRef]

34. Escudero, J.; Abasolo, D.; Hornero, R.; Espino, P.; Lopez, M. Analysis of electroencephalograms in Alzheimer's disease patients with multiscale entropy. Physiol. Meas. 2006, 27, 1091-1106. [CrossRef] [PubMed]

35. Pfeiffer, E.A. Short portable mental status questionnaire for the assessment of organic brain deficit in elderly patients. J. Am. Geriatr. Soc. 1975, 23, 433-441. [CrossRef]

36. Berg, K.; Wood-Dauphinee, S.; Williams, J.I. The Balance Scale: reliability assessment with elderly residents and patients with an acute stroke. Scand. J. Rehabil. Med. 1995, 27, 27-36. [PubMed]

37. Barry, E.; Galvin, R.; Keogh, C.; Horgan, F.; Fahey, T. Is the Timed Up and Go test a useful predictor of risk of falls in community dwelling older adults: A systematic review and meta-analysis. BMC geriatr. 2014, 14. [CrossRef] [PubMed] 
38. Mathias, S.; Nayak, U.S.; Isaacs, B. Balance in elderly patients: The "get-up and go" test. Arch. Phys. Med. Rehabil. 1986, 67, 387-389. [PubMed]

39. Fischer, B.L.; Gleason, C.E.; Gangnon, R.E.; Janczewski, J.; Shea, T.; Mahoney, J.E. Declining cognition and falls: role of risky performance of everyday mobility activities. Phys. Ther. 2014, 94, 355-362. [CrossRef] [PubMed]

40. Lin, C.H.; Liao, K.C.; Pu, S.J.; Chen, Y.C.; Liu, M.S. Associated factors for falls among the community-dwelling older people assessed by annual geriatric health examinations. PLoS ONE 2011, 6, e18976. [CrossRef] [PubMed]

41. Moe-Nilssen, R. A new method for evaluating motor control in gait under real life environmental conditions. Part 1: The instrument. Clin. Biomech. 1998, 13, 320-327. [CrossRef]

42. Karthikeyan, G.; Sheikh, S.G.; Chippala, P. Test-Retest Reliability of Short Form of Berg Balance Scale in Elderly People. Glob. Adv. Res. J. 2012, 1, 139-144.

43. Kim, S.G.; Kim, M.K. The intra- and inter-rater reliabilities of the Short Form Berg Balance Scale in institutionalized elderly people. J. Phys. Ther. Sci. 2015, 27, 2733-2734. [CrossRef] [PubMed]

44. İlçin, N.; Gürpınar, B.; Bayraktar, D.; Savcı, S.; Çetin, P.; Sarı, İ.; Akkoç, N. Cross-cultural adaptation and validation of the Turkish version of the pain catastrophizing scale among patients with ankylosing spondylitis. J. Phys. Ther. Sci. 2016, 28, 298-303. [CrossRef] [PubMed]

45. Li, Z.; Liang, Y.Y.; Wang, L.; Sheng, J.; Ma, S.J. Reliability and validity of center of pressure measures for balance assessment in older adults. J. Phys. Ther. Sci. 2016, 28, 1364-1367. [CrossRef] [PubMed]

46. Shumway-Cook, A.; Brauer, S.; Woollacott, M. Predicting the probability for falls in community dwelling older adults using the Timed Up and Go test. Phys. Ther. 2000, 80, 896-903. [PubMed]

47. Lindsay, R.; James, E.L.; Kippen, S. The Timed Up and Go Test: Unable to predict falls on the acute medical ward. Aust. J. Physiother. 2004, 50, 249-251. [CrossRef]

48. Kwoka, B.C.; Clarkb, R.A.; Pua, Y.H. Novel use of the Wii Balance Board to prospectively predict falls in community-dwelling older adults. Clin. Biomech. 2015, 30, 481-484. [CrossRef] [PubMed]

49. Tiedemann, A.; Shimada, H.; Sherrington, C.; Murray, S.; Lord, S. The comparative ability of eight functional mobility tests for predicting falls in community-dwelling older people. Age Ageing 2008, 37, 430-435. [CrossRef] [PubMed]

50. Manckoundia, P.; Mourey, F.; Pfitzenmeyer, P.; Papaxanthis, C. Comparison of motor strategies in sit-to-stand and back-to-sit motions between healthy and Alzheimer's disease elderly subjects. Neuroscience 2006, 137, 385-392. [CrossRef] [PubMed]

51. Hong, C.H.; Jiang, B.C. Multi-scale entropy approach to physiological fatigue during long-term Web browsing. Hum. Factor Ergon. Manuf. Serv. Ind. 2009, 19, 478-493. [CrossRef]

52. Lee, C.H.; Sun, T.L. Multi-Scale Entropy Analysis of Body Sway for Investigating Balance Ability during Exergame Play under Different Parameter Settings. Entropy 2015, 17, 7608-7627. [CrossRef]

53. Gao, J.B.; Hu, J.; Tung, W.W. Complexity measures of brain wave dynamics. Cogn. Neurodyn. 2011, 5, 171-182. [CrossRef] [PubMed]

54. Jiang, B.C.; Yang, W.H.; Shieh, J.S.; Fan, J.S.Z.; Peng, C.K. Entropy-Based Method for COP Data Analysis. Theor. Issues Ergon. Sci. 2013, 14, 227-246. [CrossRef]

55. Emery, C.A.; Cassidy, J.D.; Klassen, T.P.; Rosychuk, R.J.; Rowe, B.B. Development of a clinical static and dynamic standing balance measurement tool appropriate for use in adolescents. Phys. Ther. 2005, 85, 502-514. [PubMed]

56. Manor, B.; Costa, M.D.; Hu, K.; Newton, E.; Starobinets, O.; Kang, H.G.; Peng, C.K.; Novak, V.; Lipsitz, L.A. Physiological complexity and system adaptability: Evidence from postural control dynamics of older adults. J. Appl. Phys. 2010, 109, 1786-1791. [CrossRef] [PubMed]

57. Gruber, A.H.; Busa, M.A.; Gorton, G.E., III; van Emmerik, R.E.A.; Masso, P.D.; Hamill, J. Time-to-contact and multiscale entropy identify differences in postural control in adolescent idiopathic scoliosis. Gait Posture 2011, 34, 13-18. [CrossRef] [PubMed] 
58. Busa, M.A.; Gruber, A.H.; Gorton, G.E., III; Masso, P.D.; Hamill, J.; van Emmerik, R.E.A. Multiscale Entropy Identifies Complexity Changes in Postural Control of Adolescent Idopathic Scoliosis. In Proceedings of the 34th Annual International Conference of the American Society of Biomechanics, Providence, RI, USA, 18-21 August 2010.

59. Duarte, M.; Sternad, D. Complexity of human postural control in young and older adults during prolonged standing. Exp. Brain Res. 2008, 191, 265-276. [CrossRef] [PubMed]

(C) 2016 by the authors; licensee MDPI, Basel, Switzerland. This article is an open access article distributed under the terms and conditions of the Creative Commons Attribution (CC-BY) license (http://creativecommons.org/licenses/by/4.0/). 